\title{
QC Slide Review
}

National Cancer Institute

\section{Source}

National Cancer Institute. QC Slide Review. NCI Thesaurus. Code C162621.

Tissue Source Sites (TSS) will perform a QC review of tumor biospecimens by review of slides from corresponding FFPE QC blocks. For each tumor segment, an H\&E-stained slide is to be prepared and reviewed by the TSS pathologist to confirm accuracy of tissue diagnosis and tumor composition. 